\title{
Constancy of $\varphi$-holomorphic sectional curvature of an indefinite Sasaki-like almost contact manifold with $B$-metric
}

\author{
Anu Devgan and R. K. Nagaich \\ Department of Mathematics, Punjabi University, Patiala-147002, India \\ Received: 1 January 2017, Accepted: 22 July 2018 \\ Published online: 10 December 2018.
}

\begin{abstract}
The aim of the present paper is to establish a criterion for an indefinite Sasaki-like almost contact manifold with $B$-metric to reduce to a space of $\varphi$-holomorphic sectional curvature.
\end{abstract}

Keywords: Sasaki-like almost contact manifolds, $B$-metric, $\varphi$-holomorphic Sectional curvature.

\section{Introduction}

Ganchev et al. [3] defined the odd-dimensional version of almost complex manifolds with Norden metric [8,4,2] known as the almost contact manifolds with $B$-metric (Norden metric). Later, Ivanov et al. [5] introduced a new class of almost contact manifolds with $B$-metric namely Sasaki-like almost contact Complex Riemannian manifolds with $B$-metric, which is analogue to indefinite Sasakian manifold.

Tanno [9] proved the following result for an almost Hermitian manifold $\left(M^{2 n}, g, J\right)$ to reduce to a space of constant holomorphic sectional curvature.

Theorem 1. [9] Let dimension $(2 n \geq 4)$, assume that almost Hermitian manifold $\left(M^{2 n}, g, J\right)$ satisfies

$$
R(J X, J Y, J Z, J X)=R(X, Y, Z, X)
$$

for every tangent vectors $X, Y$ and $Z$. Then $\left(M^{2 n}, g, J\right)$ is of constant holomorphic sectional curvature at $x$, if and only if,

$$
R(X, J X) X \quad \text { is proportional to } \quad J X
$$

for every tangent vector $X$ at $x$ in $M$.

Tanno [9] also extended the above Theorem 1 for the Sasakian manifolds as follows.

Theorem 2. [9] A Sasakian manifold $\left(M^{2 n+1}, \phi, \eta, \xi, g\right)$ of dimension $\geq 5$, is of constant $\phi$-sectional curvature if and only if

$$
R(X, \phi X) X \quad \text { is proportional to } \phi X
$$

for every vector field $X$ such that $g(X, \xi)=0$, where $\xi$ is a characteristic vector field of $M$. 
Further, Nagaich [7] generalized the Theorem 1 for an indefinite almost Hermitian manifold and provided the following characterization.

Theorem 3. Let $\left(M^{2 n}, g, J\right)$ of dimension $2 n$, where $(n \geq 2)$ be an indefinite almost Hermitian manifold satisfying (1). Then $M$ is of constant holomorphic sectional curvature at $p$, if and only if,

$$
R(X, J X) X \quad \text { is proportional to } J X
$$

for every tangent vector $X$ at $p \in M$.

And later, Kumar et al.[6] proved the generalized version of the Theorem 2 for an indefinite Sasakian manifold as follows.

Theorem 4. Let $\left(M^{2 n+1}, \phi, \eta, \xi, g\right)(2 n \geq 4)$ be an indefinite Sasakian manifold. Then $M$ is of constant $\phi$-sectional curvature if and only if

$$
R(X, \phi X) X \quad \text { is proportional to } \phi X
$$

for every tangent vector field $X$ such that $g(X, \xi)=0$, where $\xi$ is a characteristic vector field of $M$.

Recently, we have generalized the Theorem 3 to the setting of an almost complex manifold with Norden metric as

Theorem 5. [1] Let $\left(M^{2 n}, g, J\right)(2 n \geq 4)$ be an indefinite almost complex manifold with Norden metric satisfying (1). Then $\left(M^{2 n}, g, J\right)$ is of constant holomorphic sectional curvature at $p$ if and only if

$$
R(X, J X) X \quad \text { is proportional to } \quad \alpha X+\beta J X
$$

where $\alpha$ and $\beta$ are the functions of holomorphic sectional curvature $H(X)$, for every tangent vector $X$ at $p \in M$.

In this paper, we have extended the Theorem 5 to the setting of a Sasaki-like almost contact manifold with $B$-metric to reduce to a space of constant $\varphi$-holomorphic sectional curvature.

Theorem 6. Let $\left(\bar{M}^{2 n+1}, \varphi, \zeta, \eta\right)$ be an indefinite Sasaki-like almost contact manifold with B-metric. Then $\bar{M}$ is of constant $\varphi$-holomorphic sectional curvature if and only if

$$
R(X, \varphi X) X \quad \text { is proportional to } \quad \gamma X+\delta \varphi X
$$

where $\gamma$ and $\delta$ are the functions of $\varphi$-holomorphic sectional curvature $H(X)$, for every tangent vector $X$ such that $g(X, \zeta)=0$, where $\zeta$ is a characteristic vector field of $\bar{M}$.

\section{Preliminaries}

\subsection{Almost contact manifold with B-metric}

Let $\left(\bar{M}^{2 n+1}, \varphi, \zeta, \eta\right)$ be an almost contact manifold with $B$-metric $\bar{g}$, i.e., $\bar{M}$ is a $(2 n+1)$-dimensional smooth manifold endowed with an almost contact structure $(\varphi, \zeta, \eta)$ and equipped with a pseudo-Riemannian metric $\bar{g}$, such that the following relations are satisfied [3],

$$
\begin{gathered}
\varphi \zeta=0, \quad \eta(\zeta)=1, \\
\eta(X)=\bar{g}(X, \zeta), \quad \eta(\varphi X)=0,
\end{gathered}
$$




$$
\begin{gathered}
\bar{g}(\varphi X, \varphi Y)=-\bar{g}(X, Y)+\eta(X) \eta(Y), \\
\bar{g}(\varphi X, Y)=\bar{g}(X, \varphi Y), \\
\overline{\tilde{g}}(X, Y)=\bar{g}(\varphi X, Y)+\eta(X) \eta(Y),
\end{gathered}
$$

for arbitrary tangent vector fields $X, Y \in T \bar{M}$, where $\overline{\tilde{g}}$ is called the associated metric of $\bar{g}$ on $\bar{M}$ and is also a $B$-metric on $\bar{M}$. Moreover, the manifold $(\bar{M}, \varphi, \zeta, \eta, \overline{\tilde{g}})$ is also called an almost contact manifold with $B$-metric. Infact, both $\bar{g}$ and $\overline{\tilde{g}}$ are indefinite metrics having signature $(n+1, n)$.

Let $\bar{\nabla}$ and $\bar{\nabla}$ be the Levi-Civita connections of $\bar{g}$ and $\overline{\tilde{g}}$, respectively on $\bar{M}$. In [3], the tensor field $F$ of type $(0,3)$ is defined on $\bar{M}$ as follows

$$
F(X, Y, Z)=\bar{g}\left(\left(\bar{\nabla}_{X} \varphi\right) Y, Z\right)
$$

and the following properties hold in general [3] :

$$
F(X, Y, Z)=F(X, Z, Y)=F(X, \varphi Y, \varphi Z)+\eta(Y) F(X, \zeta, Z)+\eta(Z) F(X, Y, \zeta),
$$

for any $X, Y, Z \in T \bar{M}$. The relations of $F$ with $\bar{\nabla} \zeta$ and $\bar{\nabla} \eta$ are given by :

$$
\left(\bar{\nabla}_{X} \eta\right) Y=g\left(\bar{\nabla}_{X} \zeta, Y\right)=F(X, \varphi Y, \zeta), \quad \eta\left(\bar{\nabla}_{X} \zeta\right)=0, \quad \varphi\left(\bar{\nabla}_{X} \varphi\right) \zeta=\bar{\nabla}_{X} \zeta
$$

In [3], Ganchev et al. defined eleven basic classes $F_{i}(i=1,2, \ldots .11)$ of almost contact manifolds with $B$-metric and classified the almost contact manifolds with $B$-metric in terms of the tensor $F$. The intersection of these basic classes is the class $F_{0}$, which is analogue to Kaehler manifold with Norden metric and is determined by the condition

$$
F(X, Y, Z)=0(\bar{\nabla} \varphi=\bar{\nabla} \eta=\bar{\nabla} \zeta=0) .
$$

Definition 1. [5] An almost contact manifold $(\bar{M}, \varphi, \zeta, \eta, \bar{g})$ with $B$ - metric is called Sasaki-like if the structure tensors $(\varphi, \zeta, \eta, \bar{g})$ satisfy the following equalities

$$
\begin{gathered}
F(X, Y, Z)=F(\zeta, Y, Z)=F(\zeta, \zeta, Z)=0, \\
F(X, Y, \zeta)=-\bar{g}(X, Y) .
\end{gathered}
$$

Also, the covariant derivative $\bar{\nabla} \varphi$ satisfies the following equality

$$
\left(\bar{\nabla}_{X} \varphi\right) Y=-\bar{g}(X, Y) \zeta-\eta(Y) X+2 \eta(X) \eta(Y) \zeta .
$$

A non-zero tangent vector field $U$ is classified in the following types

(i) spacelike if $\bar{g}(U, U)>0$,

(ii) timelike if $\bar{g}(U, U)<0$,

(iii) null (lightlike) if $\bar{g}(U, U)=0, U \neq 0$. 


\subsection{Curvature properties}

Let the curvature tensor $R$ of $\bar{\nabla}$ on $\bar{M}$ is given by

$$
R(X, Y) Z=\bar{\nabla}_{X} \bar{\nabla}_{Y} Z-\bar{\nabla}_{Y} \bar{\nabla}_{X} Z-\bar{\nabla}_{[X, Y]} Z
$$

The corresponding curvature $(0,4)$-tensor with respect to $\bar{g}$ is given by

$$
R(X, Y, Z, W)=\bar{g}(R(X, Y) Z, W)
$$

and satisfies the following properties

$$
\begin{gathered}
R X, Y, Z, W)=-R(Y, X, Z, W)=-R(X, Y, W, Z), \\
R(X, Y, Z, W)+R(Y, Z, X, W)+R(Z, X, Y, W)=0, \\
R(X, Y, Z, W)=-R(X, Y, \varphi Z, \varphi W)
\end{gathered}
$$

for all tangent vector fields $X, Y, Z$ and $W$ on $\bar{M}$.

The associated curvature tensor $\tilde{R}$ of $\tilde{\bar{\nabla}}$ on $\bar{M}$ is defined as

$$
\tilde{R}(X, Y, Z, W)=R(X, Y, Z, \varphi W)
$$

Thus, for the curvature tensor $R$, we have

$$
R(X, Y, Z, \varphi W)=R(X, Y, \varphi Z, W)
$$

Let $\alpha$ denote a non-degenerate 2-plane in the tangent space $T_{p} \bar{M}$. Then the sectional curvature for $\alpha$ with respect to $\bar{g}$ and $R$ is given by

$$
K(\alpha, p)=\frac{R(U, V, U, V)}{\bar{g}(U, U) \bar{g}(V, V)-\bar{g}(U, V)^{2}} .
$$

where $\{U, V\}$ is an orthogonal basis of $\alpha$ and $p \in \bar{M}$.

Definition 2. A 2-plane $\alpha=\{U, \varphi U\}$, where $U$ is orthonormal to $\zeta$ is known as $\varphi$-holomorphic section (respectively, a $\zeta$-section) if $\alpha=\varphi \alpha$ (respectively, $\zeta \in \alpha$ ) and the curvature associated with this is said to be $\varphi$-holomorphic sectional curvature, denoted by $H(U)$ and given as

$$
H(U)=\frac{R(U, \varphi U, U, \varphi U)}{\bar{g}(U, U) \bar{g}(\varphi U, \varphi U)-\bar{g}(U, \varphi U)^{2}}
$$

Moreover, if $H(U)$ is always constant with respect to every unit tangent vector $U \in T \bar{M}$, then $\bar{M}$ is said to be of constant $\varphi$-holomorphic sectional curvature or a Sasakian space form.

\subsection{Sasaki-like almost contact manifold with B-metric}

In [5], Ivanov defined the odd dimensional version of an indefinite Kaehler manifold known as Sasaki-like almost contact manifold with $B$-metric and proved the following result. 
Lemma 1. [5] For a Sasaki-like almost contact manifold $(\bar{M}, \varphi, \zeta, \eta, \bar{g})$ with B-metric the next formula holds

$$
\begin{aligned}
R(X, Y, \varphi Z, \varphi U)-R(X, Y, Z, \varphi U) & =\{\bar{g}(Y, Z)-2 \eta(Y) \eta(Z)\} \bar{g}(X, \varphi U)+\{\bar{g}(Y, U)-2 \eta(Y) \eta(U)\} \bar{g}(X, \varphi Z) \\
& -\{\bar{g}(X, Z)-2 \eta(X) \eta(Z)\} \bar{g}(Y, \varphi U)-\{\bar{g}(X, U)-2 \eta(X) \eta(U)\} \bar{g}(Y, \varphi Z) .
\end{aligned}
$$

In particular, we have

$$
R(X, Y) \zeta=\eta(Y) X-\eta(X) Y
$$

and

$$
R(\zeta, X) \zeta=-X
$$

The equation (21) further implies

$$
\begin{aligned}
R(X, Y) \varphi Z= & \varphi R(X, Y) Z-2 \varphi \eta(Z) R(X, Y) \zeta-\bar{g}(X, Z) \varphi Y+\bar{g}(X, \varphi Z) Y \\
& -\bar{g}(Y, \varphi Z) X+2\{\bar{g}(Y, \varphi Z) \eta(X)-\bar{g}(X, \varphi Z) \eta(Y)\} \zeta
\end{aligned}
$$

Replacing $Y$ by $\varphi X$ and $Z$ by $\varphi X$ in above equation (23) and use of (22) yields,

$$
\left.R(X, \varphi X) X=-\left\{R(X, \varphi X) \varphi X+(\eta(X))^{2} \varphi X+2 \bar{g}(X, \varphi X) X+2 \bar{g}(\varphi X, \varphi X) \varphi X-3 \bar{g}(X, \varphi X) \eta(X)\right\} \zeta\right\}
$$

\section{Constancy of $\varphi$-holomorphic sectional curvature}

Now we will prove the main result.

Proof. Initially assume that $\bar{M}$ be an indefinite Sasaki-like almost contact manifold with $B$-metric, then using formula (20), we obtain

$$
R(X, \varphi X) X=-H(X) \rho X+H(X) \varphi X .
$$

where $X$ denotes a unit tangent vector such that $\bar{g}(X, \varphi X)=\rho(\neq 0)$. By using the fact that $\bar{M}$ is having constant $\varphi$-holomorphic sectional curvature and the equation (25), the necessity of the assertion follows. To prove the converse part, the following two cases have been considered.

Case I. For the space-like,or in other words, $\bar{g}(X, X)=\bar{g}(Y, Y)$. Let $\{X, Y\}$ denote an orthonormal pair of vectors in $\bar{M}$ such that

$$
\begin{gathered}
\bar{g}(X, X)=-\bar{g}(\varphi X, \varphi X)=1, \\
\bar{g}(Y, Y)=-\bar{g}(\varphi Y, \varphi Y)=1, \\
\bar{g}(X, \varphi X)=\bar{g}(Y, \varphi Y)=\rho(\neq 0)
\end{gathered}
$$

and

$$
\bar{g}(X, \varphi Y)=\bar{g}(\varphi X, Y)=0 .
$$

In this case, $X^{* *}$ and $Z^{* *}$ be defined by

$$
X^{* *}=\cos \theta X+\sin \theta Y
$$

and

$$
Z^{* *}=-\sin \theta X+\cos \theta Y .
$$


Clearly, $\left\{X^{* *}, Z^{* *}\right\}$ also form an orthonormal pair of vectors in $\bar{M}$ and using the above relation (7), we have

$$
R\left(X^{* *}, \varphi X^{* *}\right) X^{* *} \sim \gamma X^{* *}+\delta \varphi X^{* *} .
$$

Taking inner product of above equation with $\varphi Z^{* *}$, we have

$$
R\left(X^{* *}, \varphi X^{* *}, X^{* *}, \varphi Z^{* *}\right)=0
$$

Also, by using the linear properties of Riemannian curvature tensor $R$, we obtain

$$
\cos \theta \sin \theta\left\{-\cos ^{2} \theta R(X, \varphi X, X, \varphi X)+\sin ^{2} \theta R(Y, \varphi Y, Y, \varphi Y)+\left(\cos ^{2} \theta-\sin ^{2} \theta\right) R(Y, \varphi Y, X, \varphi X)\right\}=0 .
$$

Considering $\theta=\frac{\pi}{4}$ yields,

$$
H(X)=H(Y) .
$$

If $\{Z, W\}$ is a $\varphi$-holomorphic section then $\varphi Z=p Z+q Z$, for any scalars $p$ and $q$. Thus, $\{Z, \varphi Z\}=\{Z, p Z+q Z\}=\{Z, W\}$ and similarly $\{W, \varphi W\}=\{Z, W\}$. therefore $\{Z, \varphi Z\}=\{W, \varphi W\}$ and hence $H(Z)=H(W)$.

On the contrary if $\{Z, W\}$ is not a $\varphi$-holomorphic section then there must exist unit vectors $X \in\{Z, \varphi Z\}^{\perp}$ and $Y \in$ $\{W, \varphi W\}^{\perp}$ that determine a $\varphi$-holomorphic section $\{X, Y\}$ and thus, we have

$$
H(Z)=H(X)=H(Y)=H(W),
$$

which proves that any $\varphi$-holomorphic section has the same $\varphi$-holomorphic sectional curvature.

Now, let the $\operatorname{dim}(\bar{M})=5$ and using the properties of curvature tensor $R$, the following relations hold.

$$
\begin{aligned}
& R(X, \varphi X) X=H(X)\{-\rho X+\varphi X\} \\
& R(X, \varphi X) Y=\frac{1}{1+\rho^{2}}\{R(X, \varphi X, Y, \varphi Y)(\rho Y-\varphi Y)\} \\
& R(X, \varphi Y) X=\frac{1}{1+\rho^{2}}\{R(X, \varphi Y, X, Y)(Y+\rho \varphi Y)+R(X, \varphi Y, X, \varphi Y)(\rho Y-\varphi Y)\} \\
& R(Y, \varphi X) X=\frac{1}{1+\rho^{2}}\{R(Y, \varphi X, X, Y)(Y+\rho \varphi Y)+R(Y, \varphi X, X, \varphi Y)(\rho Y-\varphi Y)\} \\
& R(X, \varphi Y) Y=\frac{1}{1+\rho^{2}}\{R(X, \varphi Y, Y, X)(X+\rho \varphi X)+R(X, \varphi Y, Y, \varphi X)(\rho X-\varphi X)\} \\
& R(Y, \varphi X) Y=\frac{1}{1+\rho^{2}}\{R(Y, \varphi X, Y, X)(X+\rho \varphi X)+R(Y, \varphi X, Y, \varphi X)(\rho X-\varphi X)\} \\
& R(Y, \varphi Y) X=\frac{1}{1+\rho^{2}}\{R(Y, \varphi Y, X, \varphi X)(\rho X-\varphi X)\} \\
& R(Y, \varphi Y) Y=H(Y)\{-\rho Y+\varphi Y\} .
\end{aligned}
$$

Now, define $X^{* *}=d X+e Y$ where $d^{2}+e^{2}=1$, then making use of the above algebraic relations (28), we have

$$
R\left(X^{* *}, \varphi X^{* *}\right) X^{* *}=E_{1} X+E_{2} Y+E_{3} \varphi X+E_{4} \varphi Y,
$$

where

$$
E_{3}=d^{3} H(X)-\frac{d e^{2}}{\left(1+\rho^{2}\right)} E_{5}, \quad E_{4}=e^{3} H(X)-\frac{d^{2} e}{\left(1+\rho^{2}\right)} E_{5},
$$


and

$$
E_{5}=R(X, \varphi Y, Y, \varphi X)+R(Y, \varphi X, Y, \varphi X)+R(Y, \varphi Y, X, \varphi X) .
$$

On the other hand, equation (27) yields,

$$
R\left(X^{* *}, \varphi X^{* *}\right) X^{* *}=H\left(X^{* *}\right)\left\{-\rho X^{* *}+\varphi X^{* *}\right\}=H\left(X^{* *}\right)\{\rho d X+\rho e Y-d \varphi X-e \varphi Y\} .
$$

Comparing the equations (29) and (30), we obtain

$$
d^{2} H(X)-\frac{e^{2}}{\left(1+\rho^{2}\right)} E_{5}=H\left(X^{* *}\right), \quad e^{2} H(X)-\frac{d^{2}}{\left(1+\rho^{2}\right)} E_{5}=H\left(X^{* *}\right),
$$

upon solving the above equations, we have

$$
E_{5}=-\left(1+\rho^{2}\right) H(X)
$$

and hence consequently

$$
H\left(X^{* *}\right)=\left(d^{2}+e^{2}\right) H(X)=H(X) .
$$

Similarly, on the parallel lines, we prove that

$$
H\left(Y^{* *}\right)=H(Y) .
$$

Thus, we have proved that the manifold $\bar{M}$ is of constant $\varphi$-holomorphic sectional curvature.

Case II: When the metric is timelike, or in other words, $\bar{g}(X, X)=-\bar{g}(Y, Y)$, where either $X$ and $Y$ are spacelike and timelike vectors, respectively or vice versa. Let $\{X, Y\}$ denote a pair of orthonormal vectors in $\bar{M}$ such that

$$
\begin{gathered}
\bar{g}(X, X)=-\bar{g}(\varphi X, \varphi X)=1, \\
\bar{g}(Y, Y)=-\bar{g}(\varphi Y, \varphi Y)=-1, \\
\bar{g}(X, \varphi X)=-\bar{g}(Y, \varphi Y)=\rho(\neq 0)
\end{gathered}
$$

and

$$
\bar{g}(X, \varphi Y)=\bar{g}(\varphi X, Y)=0 .
$$

Further, we define $X^{\prime \prime}$ and $Z^{\prime \prime}$ by

$$
X^{\prime \prime}=\cosh \theta X+\sinh \theta Y
$$

and

$$
Z^{\prime \prime}=-\sinh \theta \varphi X+\cosh \theta \varphi Y
$$

then $X^{\prime \prime}, Z^{\prime \prime}$ form an orthonormal pair of vectors in $\bar{M}$ and therefore making use of the relation (7), we have

$$
R\left(X^{\prime \prime}, \varphi X^{\prime \prime}\right) X^{\prime \prime} \sim \gamma X^{\prime \prime}+\delta \varphi X^{\prime \prime} .
$$

Taking inner product of above equation with $Z^{\prime \prime}$, we obtain,

$$
R\left(X^{\prime \prime}, \varphi X^{\prime \prime}, X^{\prime \prime}, Z^{\prime \prime}\right)=0
$$

further, using the linearity properties of curvature tensor, we have

$$
\cosh \theta \sinh \theta\left\{\cos ^{2} h \theta H(X)-\sin ^{2} h \theta H(Y)-\left(\cos ^{2} h \theta-\sin ^{2} h \theta\right) R(X, \varphi X, Y, \varphi Y)\right\}=0 .
$$


Considering $\theta=\frac{i \pi}{4}$, we get

$$
H(X)=H(Y) .
$$

Further, using the same argument given in Case I, we obtain that any holomorphic section has same sectional curvature. Now, assuming the $\operatorname{dim}(\bar{M})=5$ and using the curvature properties of curvature tensor $R$, we have the following relations

$$
\begin{aligned}
& R(X, \varphi X) X=-H(X)\{\rho X-\varphi X\} \\
& R(X, \varphi X) Y=\frac{1}{1+\rho^{2}}\{R(X, \varphi X, Y, \varphi Y)(-\rho Y+\varphi Y)\} \\
& R(X, \varphi Y) X=\frac{1}{1+\rho^{2}}\{R(X, \varphi X, Y, \varphi Y)(-\rho Y+\varphi Y)\} \\
& R(X, \varphi Y) X=\frac{1}{1+\rho^{2}}\{R(X, \varphi Y, X, Y)(-Y-\rho \varphi Y)+R(X, \varphi Y, X, \varphi Y)(-\rho Y+\varphi Y)\} \\
& R(Y, \varphi X) X=\frac{1}{1+\rho^{2}}\{R(Y, \varphi X, X, Y)(-Y-\rho \varphi Y)+R(Y, \varphi X, X, \varphi Y)(-\rho Y+\varphi Y)\} \\
& R(X, \varphi Y) Y=\frac{1}{1+\rho^{2}}\{R(X, \varphi Y, Y, X)(X+\rho \varphi X)+R(X, \varphi Y, Y, \varphi X)(\rho X-\varphi X)\} \\
& R(Y, \varphi X) Y=\frac{1}{1+\rho^{2}}\{R(Y, \varphi X, Y, X)(X+\rho \varphi X)+R(Y, \varphi X, Y, \varphi X)(\rho X-\varphi X)\} \\
& R(Y, \varphi Y) X=\frac{1}{1+\rho^{2}}\{R(Y, \varphi Y, X, \varphi X)(\rho X-\varphi X)\} \\
& R(Y, \varphi Y) Y=-H(Y)\{-\rho Y+\varphi Y\} .
\end{aligned}
$$

Now, define $X^{\prime \prime}=d X+e Y$ with $d^{2}-e^{2}=1$, then using the above relations, we have

$$
R\left(X^{\prime \prime}, \varphi X^{\prime \prime}\right) X^{\prime \prime}=E_{1} X+E_{2} Y+E_{3} \varphi X+E_{4} \varphi Y
$$

where

$$
E_{3}=d^{3} H(X)\left(1+\rho^{2}\right)-\frac{d e^{2}}{\left(1+\rho^{2}\right)} E_{5}, \quad E_{4}=-e^{3} H(X)\left(1+\rho^{2}\right)+\frac{d^{2} e}{\left(1+\rho^{2}\right)} E_{5},
$$

and $E_{5}=R(X, \varphi Y, Y, \varphi X)+R(Y, \varphi X, Y, \varphi X)+R(Y, \varphi Y, X, \varphi X)$. On the other hand, using (32), we have

$$
R\left(X^{\prime \prime}, \varphi X^{\prime \prime}\right) X^{\prime \prime}=-H\left(X^{\prime \prime}\right)\{\rho d X+\rho e Y-d \varphi X-e \varphi Y\} .
$$

Comparing (34) and (35), we obtain

$$
d^{2} H(X)-\frac{e^{2}}{\left(1+\rho^{2}\right)} E_{5}=H\left(X^{\prime \prime}\right), \quad-e^{2} H(X)+\frac{d^{2}}{\left(1+\rho^{2}\right)} E_{5}=H\left(X^{\prime \prime}\right)
$$

on solving these equations, we obtain

$$
E_{5}=\left(1+\rho^{2}\right) H(X)
$$

and consequently

$$
H\left(X^{\prime \prime}\right)=\left(d^{2}-e^{2}\right) H(X)=H(X)
$$

Similarly, we can prove

$$
H\left(Y^{\prime \prime}\right)=H(Y) .
$$

Thus, the manifold $\bar{M}$ is of constant $\varphi$-holomorphic sectional curvature. 
Hence, we conclude that Theorem 2 can be derived by considering $g(X, \varphi X)=\rho=0$, in Theorem 6 .

Similarly, by taking $g(X, \varphi X)=\rho=0$, in Theorem 6 , the constancy of $\varphi$-holomorphic sectional curvature can be derived for an indefinite almost Sasakian manifold with some minor changes and thus, Theorem 6 provides a generalization of Theorem 4 .

\section{Competing interests}

The authors declare that they have no competing interests.

\section{Authors' contributions}

All authors have contributed to all parts of the article. All authors read and approved the final manuscript.

\section{References}

[1] A. Devgan and R. K. Nagaich, Characterization theorem for an indefinite almost complex manifold with Norden metric, International Journal of Pure and Applied Mathematics, 114(4) (2017), 857-866.

[2] G. Ganchev, A. Borisov, Note on the almost complex manifolds with a Norden metric, Compt. Rend. Acad. Bulg. Sci., 39 (1986), 31-34.

[3] G. Ganchev, V. Mihova, K. Gribachev, Almost contact manifolds with B-metric, Math. Balkanica (N.S.), 7(3-4) (1993), 261-276.

[4] K. I. Gribachev, D. G. Mekerov, G. D. Dzhelepov, Generalized B-manifolds, Compt. Rend. Acad. Bulg. Sci., 38(3) (1985), 299302.

[5] S. Ivanov, H. Manev and M. Manev, Sasaki-like Almost contact complex Riemannian manifolds, J. Geom and Physics, 107 (2016), 136-148.

[6] R. Kumar, R. Rani and R. K. Nagaich, Constancy of $\varphi$-holomorphic sectional curvature in indefinite Sasakian manifolds, Int. Elec. J. of Geom., 2 (2009), 34-40.

[7] R. K. Nagaich, Constancy of holopmorphic sectional curvature in indefinite almost hermition manifolds, Kodai. Math. J., 16 (1993), 327-331.

[8] A. P. Norden, On a class of four-dimensional A-spaces, Russian Math., 17(4) (1960), 145-157.

[9] S. Tanno, Constancy of holomorphic sectional curvature in almost Hermition manifolds, Kodai. Math. Sem. Rep, 25 (1973), $190-201$. 